\title{
Dual and Mixture Monte Carlo Localization Algorithms for Mobile Wireless Sensor Networks
}

\author{
Enrique Stevens-Navarro, Vijayanth Vivekanandan, and Vincent W.S. Wong \\ Department of Electrical and Computer Engineering \\ The University of British Columbia, Vancouver, Canada \\ E-mail: \{enriques, vijayv, vincentw\}@ece.ubc.ca
}

\begin{abstract}
In this paper, we consider a mobile wireless sensor network where both sensor nodes and the seeds are moving. We propose and analyze two variations of the Monte Carlo Localization (MCL) algorithms, namely: dual MCL and mixture MCL, for mobile sensor networks. We conduct simulation experiments to evaluate the performance of these two algorithms by varying the number of seeds, number of nodes, number of samples, velocity of nodes, and radio pattern degree of irregularity. Results show that both dual MCL and mixture MCL are more accurate than the original MCL algorithm. In terms of the trade off between the computational time and estimated location accuracy, the mixture MCL has a better performance than both dual MCL and the original MCL algorithms.
\end{abstract}

\section{INTRODUCTION}

Wireless sensor networks (WSNs) can be deployed in different scenarios, ranging from environment monitoring to target tracking for military applications. When an event has occurred, the sensor node needs to send a message to the central authority (e.g., sink node) by relaying through other nodes in a multi-hop manner. The message is required to indicate the location of the node which detected the event. Localization is crucial for some applications in WSNs [1].

Various localization algorithms have been proposed for WSNs recently. Some of them are centralized algorithms (e.g., [2] [3]) while some others are distributed (e.g., [4] [5]). In general, distributed algorithms are more robust and energy efficient than centralized schemes. In each group, some algorithms assume simple connectivity information between neighboring nodes (e.g., [2] [6]) while some others need to gather the ranging information (e.g., estimated distance between two neighboring nodes) [7] and angle information [8]. In order to determine the actual or absolute position of each sensor node, a small fraction of special nodes (called seeds or anchor nodes) with known positions is necessary. Each seed may be equipped with a GPS (Global Positioning System) receiver to obtain its absolute position information.

Recent localization schemes have explored the use of mobile seeds and nodes. In [9], a single mobile seed traverses the network and allows stationary sensor nodes to compute their location estimates based on at least three neighboring nodes' locations. Multiple mobile seeds are used in [10]. In [11], an extended Kalman filter-based state estimator is used in tandem with mobile robots for localization.

In [12], both seeds and sensors nodes are mobile and a Monte Carlo Localization (MCL) algorithm is proposed.
MCL is a recursive Bayes filter that estimates the posterior distribution of node's positions conditioned on sensor information. The MCL algorithm has two steps. In the prediction step, the sensor node uses a motion model to predict its possible location within a two dimensional Cartesian space based on previous samples and its movement. In the filtering step, the node uses a filtering mechanism to eliminate those predicted locations which are inconsistent with the current sensor information. Simulation results show that the MCL algorithm gives lower estimation error than both Centroid [13] and the Amorphous [14] localization algorithms.

In this paper, we extend the work in [12] [15] and propose variations of the MCL algorithms, namely: dual MCL and mixture MCL, for mobile WSNs. The dual MCL can be considered as the logical inverse of the original MCL algorithm. The mixture MCL is a combination of both the original MCL and dual MCL algorithms.

We conduct simulation experiments to evaluate the performance of these two algorithms by varying the number of seeds, number of nodes, number of samples, velocity of nodes, and radio pattern degree of irregularity. Results show that both dual MCL and mixture MCL are more accurate than the original MCL algorithm. In terms of the trade off between the computational time and estimated location accuracy, the mixture MCL has a better performance than both dual MCL and the original MCL algorithms.

This paper is organized as follows. Section II describes our proposed dual MCL and mixture MCL algorithms. The performance evaluations are presented in Section III. Conclusions are given in Section IV.

\section{DuAl And Mixture MCL Algorithms}

In this section, we first describe the network model and state the assumptions. We then present the dual MCL and mixture MCL algorithms.

\section{A. Network Model and Assumptions}

In a mobile WSN, sensor nodes and the seeds are allowed to move freely within the deployment area. As a sensor node moves within its deployment area, its previous location estimation may become obsolete. However, since the sensor node can obtain the location information from the seed nodes, it allows the sensor to update its location estimation. 
The sensor network is modeled as a dynamic discrete-time system as follows: Let $t=\{1,2, \cdots$,$\} denote the time. The$ location estimation of a particular sensor node at time $t$ is denoted by $l_{t}$. The conditional probability for the motion model is denoted by $p\left(l_{t} \mid l_{t-1}\right)$. We assume the use of the following motion model:

$$
p\left(l_{t} \mid l_{t-1}\right)= \begin{cases}\frac{1}{\pi d_{\max }^{2}} & \text { if } d\left(l_{t}, l_{t-1}\right)<d_{\max } \\ 0 & \text { otherwise }\end{cases}
$$

where $d_{\text {max }}$ the maximum distance that a seed or sensor node can travel between localization steps, and $d\left(l_{t}, l_{t-1}\right)$ denotes the Euclidian distance between two locations $l_{t}$ and $l_{t-1}$.

Within a time interval $\Delta t$, a sensor node may receive the location information from the seed nodes. We denote this location information (or observation) as $o_{t}$. The perceptual model $p\left(l_{t} \mid o_{t}\right)$ describes the likelihood of the node being at location $l_{t}$ given the sensor information from seed nodes.

Although the techniques of dual MCL and mixture MCL have been applied to localization for mobile robots [15], there are two differences between localization for mobile robots and mobile sensor networks [12]:

1) A pre-determined map is usually available for robot localization, whereas the characteristics of the terrain for the deployment area of sensors are usually not known in advance.

2) A robot can control its direction of movement whereas the mobile sensors have no control of their movement. In addition, the sensors cannot obtain the information regarding their speed and direction.

The dual MCL and mixture MCL algorithms described below have been modified such that they can work effectively for mobile sensor networks. One of the modifications is that during the filtering step, each measurement is assumed to be independent, and the weight of each sample is either equal to one or zero.

\section{B. Dual MCL Algorithm}

The main idea behind the dual MCL is to invert the sampling process of the conditional densities used in the original MCL. In the dual MCL, samples are obtained from $p\left(l_{t} \mid o_{t}\right)$ and are filtered by using $p\left(l_{t} \mid l_{t-1}\right)$. The proposed dual MCL algorithm in algorithmic form is as follows:

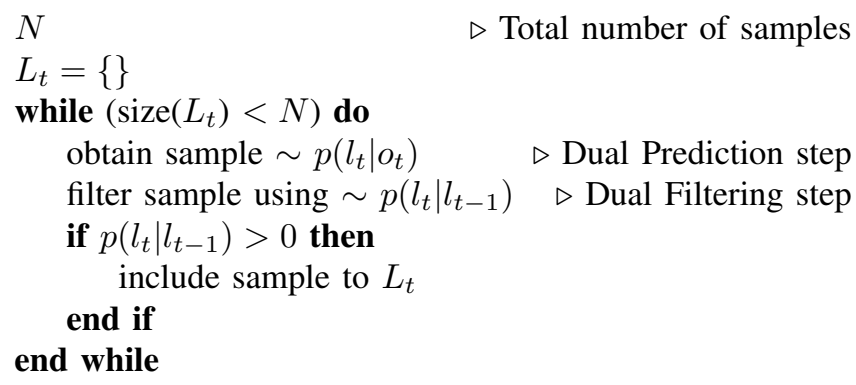

where $L_{t}$ denotes the set of possible locations at time $t$.
The dual MCL also works under the prediction and filtering steps. Due to the inversion of the sampling process, the prediction step of the dual is more elaborated than the prediction step of the original MCL. The filtering approach indicates that whether the importance weight of each sample is 0 or 1 . The posterior distribution of possible locations is approximated by a set of weighted samples. The prediction and filtering steps of the dual MCL need to be initialized. Details and considerations of such steps are described in the following subsections.

1) Dual MCL Prediction Step: To obtain a sample from $p\left(l_{t} \mid o_{t}\right)$, the dual prediction step first generates a uniform distributed sample (i.e., location) from the deployment area and verifies it as a valid sample under its current sensor information. The deployment area is defined as a bounded two dimensional Cartesian space where the location of the node is given by the $(x, y)$ coordinates. The area is bounded between 0 and $X_{\max }$, and between 0 and $Y_{\max }$. The initial location (or sample) $l_{0}$ is generated by a uniform distribution:

$$
p\left(l_{0}\right)=\frac{1}{\text { Total_Area }}=\left(\frac{1}{X_{\max }}, \frac{1}{Y_{\max }}\right)
$$

In order to obtain the sample from $p\left(l_{t} \mid o_{t}\right)$, the generated sample $l_{0}$ has to be validated by the current sensor information. We assume that at time $t$, every node within the range $r$ of a seed will be able to hear the location information from that seed. The nodes are allowed to exchange information about the seed locations heard in the previous time interval.

Similar to [12], the filter condition used to validate a sample is given by:

$$
\text { filter }(l)=\forall s \in S, d(l, s) \leq r \wedge \forall s \in T, r<d(l, s) \leq 2 r
$$

where $S$ denotes the set of all seeds heard by a node, $T$ denotes the set of all seeds heard by the node's neighbors but not by itself, and $r$ is the transmission range of a node or seed.

If the filter condition is true, the conditional probability distribution of $p\left(l_{t} \mid o_{t}\right)$ will be uniformly distributed. Otherwise, $p\left(l_{t} \mid o_{t}\right)$ will be equal to zero. Thus, if the generated sample is validated by the filter condition (3), then we have a sample from $p\left(l_{t} \mid o_{t}\right)$ with weight being equal to 1 . In other words, the location $l_{o}$ is valid under the current sensor information. This process is repeated until the dual MCL has $N$ valid predicted samples. After dual MCL has been initialized, the subsequent iterations predict the locations based on the previous locations and recent information from the seeds.

2) Dual MCL Filtering Step: For the filtering step, the validated predicted sample $l_{o}$ has to be within the set of possible locations based on $p\left(l_{t} \mid l_{t-1}\right)$. The node needs to eliminate the infeasible locations based on its previous location information. All infeasible locations are assigned a weight of 0 , whereas the valid locations are assigned a weight of 1 . The dual filtering condition can be stated as follows:

$$
\text { filter_dual }(l)=d\left(l, l_{t-1}\right) \leq d_{\max }
$$

where $l_{t-1}$ is the estimated location of the node at the previous localization step. If localization is performed every $K$ time 
units, we have:

$$
d_{\max }=K \cdot v_{\max }
$$

where $v_{\max }$ is the maximum velocity of a seed or mobile sensor. If the filter condition is true, then $p\left(l_{t} \mid l_{t-1}\right)$ will be uniformly distributed. Otherwise, $p\left(l_{t} \mid l_{t-1}\right)$ is equal to zero. After filtering, there may be less than $N$ possible locations. The prediction and filtering steps are repeated until there are at least $N$ possible locations.

\section{Mixture MCL Algorithm}

In the mixture MCL algorithm, samples are generated by both the original MCL and the dual MCL algorithms. These samples are mixed together. A mixing rate $\phi$ is defined to be between $0 \leq \phi \leq 1$. The mixture MCL generates each sample with probability $1-\phi$ by using the original MCL sampling methodology, and with probability $\phi$ by using the dual MCL sampling methodology. The mixture MCL in algorithmic form is as follows:

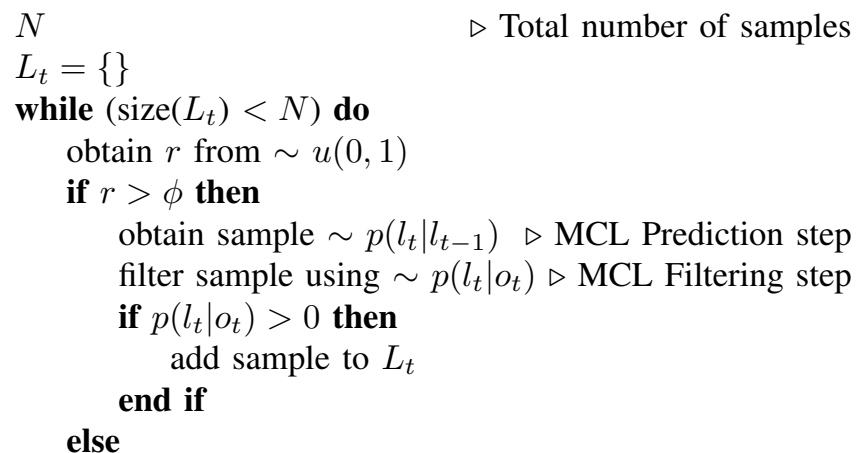
obtain sample $\sim p\left(l_{t} \mid o_{t}\right) \quad \triangleright$ Dual Prediction step filter sample using $\sim p\left(l_{t} \mid l_{t-1}\right) \quad \triangleright$ Dual Filtering

step

$$
\begin{aligned}
& \text { if } p\left(l_{t} \mid l_{t-1}\right)>0 \text { then } \\
& \text { add sample to } L_{t} \\
& \text { end if } \\
& \text { end if }
\end{aligned}
$$$$
\text { end while }
$$

Note that re-sampling is required in order to avoid degeneracy of the sampling processes. The re-sampling ensures that there is an effective sample size to represent the approximated distributions. Similar to the dual MCL algorithm, in the filtering step, the importance weight for each samples can either be 1 or 0 .

\section{PERformance Evaluation}

In this section, we compare the performance between the dual MCL, mixture MCL, and the original MCL algorithms. The localization algorithms are implemented by extending the simulator available from [16]. For all simulations, the sensor nodes are initially randomly distributed over a square area of $250 \mathrm{~m} \times 250 \mathrm{~m}$. The transmission range $r$ of both the sensor nodes and seeds is assumed to be a perfect circle with radio range of $25 \mathrm{~m}$.

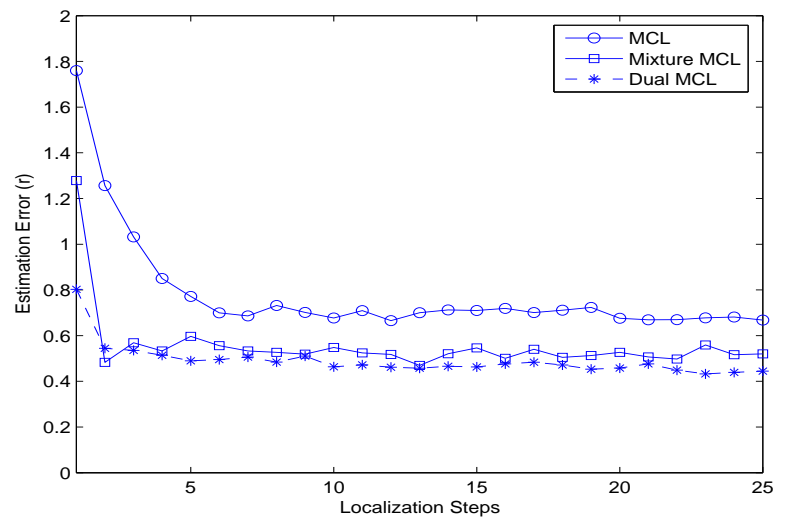

Fig. 1. Estimation error versus location convergence with $n_{d}=10, s_{d}=2$, $v_{\max }=r, N=35, K=5$ and $\phi=0.2$.

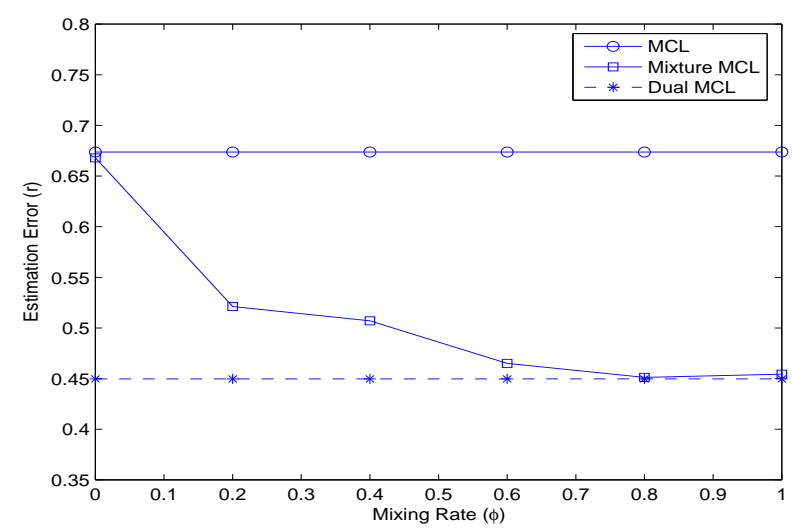

Fig. 2. Estimation error versus mixing rate $(\phi)$ with $n_{d}=10, s_{d}=2$, $v_{\max }=r, N=35$ and $K=5$.

The unit of $v_{\max }$ (the maximum velocity of a node or seed) is given in distance per time unit. Unless stated otherwise, $v_{\max }$ is set to $r$. Thus, it is equal to radio range distance per unit of time. If we assume that the unit of time is in minute, then the maximum velocity allowed for a node or seed is approximately equal to $0.42 \mathrm{~m} / \mathrm{s}$.

Both seeds and sensors use a random waypoint mobility model. Localization is performed every $K$ time units. The estimated location of a sensor node is calculated as the average of all the samples obtained by the node. The estimation error is normalized with respect to the radio range $r$.

Different values of node density $\left(n_{d}\right)$ and seed density $\left(s_{d}\right)$ are used. They are calculated as follows:

$$
n_{d}=\frac{\pi r^{2} n_{m}}{\text { TotalArea }}
$$

and

$$
s_{d}=\frac{\pi r^{2} s_{m}}{\text { TotalArea }}
$$

where $n_{m}$ is the total number of nodes, $s_{m}$ is the total number of seeds, and TotalArea is the total deployment area. As an example, a node density $n_{d}$ of 10 corresponds to 320 nodes and a seed density $s_{d}$ of 2 corresponds to 64 seeds. 


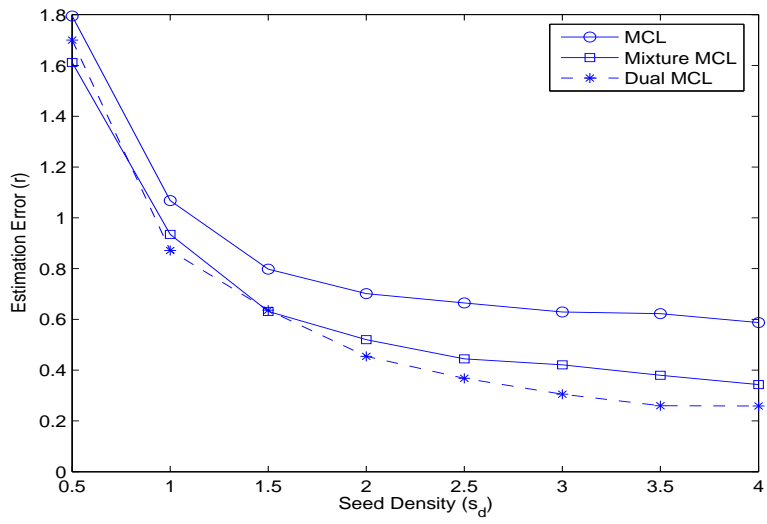

Fig. 3. Estimation error versus seed density with $n_{d}=10, v_{\max }=r$, $N=35, K=5$ and $\phi=0.2$.

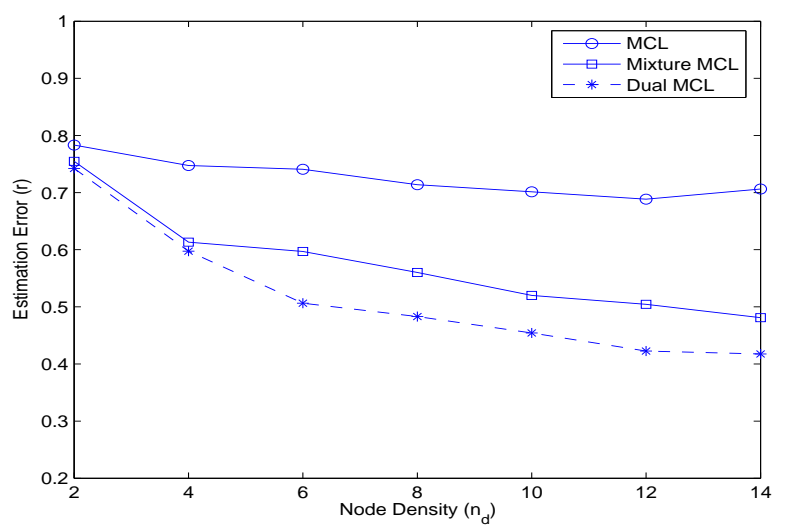

Fig. 4. Estimation error versus node density with $s_{d}=2, v_{\max }=r$, $N=35, K=5$ and $\phi=0.2$.

\section{A. Results and Discussions}

Fig. 1 shows that the transient phase is equal to approximately 8 to 10 localization steps. During the transient phase, the estimation error decreases very fast. After that, the localization process reaches a steady state. Both dual MCL and mixture MCL algorithms reach the steady state faster than the original MCL algorithm (in terms of a smaller number of localization steps). In addition, the results show that both dual MCL and mixture MCL (with $\phi=0.2$ ) algorithms provide a lower estimation errors than the original MCL. Results show that when the mixture MCL algorithm is being used, the estimation error is reduced by $22.7 \%$ when compared to the original MCL. When the dual MCL algorithm is being used, the estimation error is reduced by $33.3 \%$ when compared to the original MCL.

Fig. 2 shows the estimation error versus the mixing rate $\phi$ for the mixture MCL algorithm. Results show that for the mixture MCL with $\phi$ between 0.2 and 0.4 , it can already give a better performance than the original MCL algorithm. For the rest of this section, a mixing rate $\phi$ of 0.2 is used in the simulations for the mixture MCL.

Fig. 3 shows the estimation error versus the seed density. When the number of seeds increases, the sensor nodes can

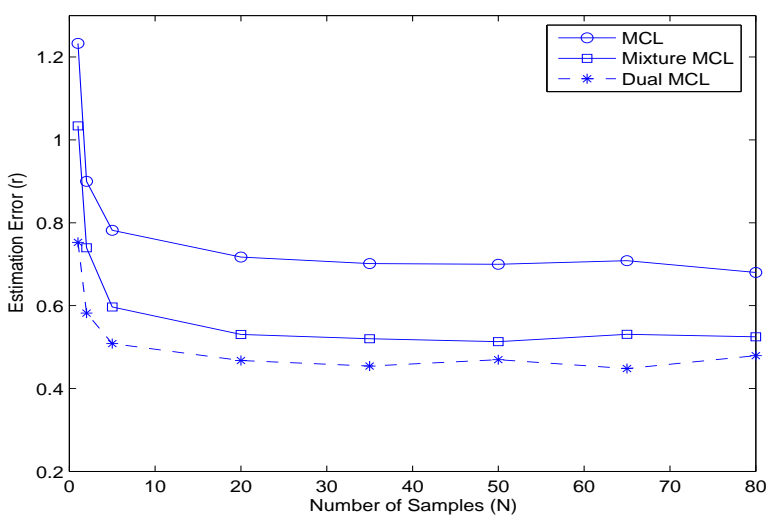

Fig. 5. Estimation error versus number of samples with $n_{d}=10, s_{d}=2$, $v_{\max }=r, K=5$ and $\phi=0.2$.

obtain more location information. However, since each seed requires a GPS receiver, it increases the deployment cost. Fig. 3 shows that for both dual and mixture MCL algorithms, the further reduction of the estimation error is insignificant when $s_{d}$ is greater than 3.5.

Fig. 4 shows the estimation error versus the node density. Similar to the results in [12], the performance of the original MCL algorithm remains the same when the node density increases. However, this is not the case for dual MCL and mixture MCL algorithms. As node density increases, the accuracy of both schemes also increases. This is because the accuracy of the dual and mixture MCL algorithms also depends on the information from the neighboring sensors to determine the valid samples. A higher node density provides more information and thus improves the performance.

Fig. 5 shows the estimation error versus the number of samples $N$. Maintaining more samples can improve the accuracy up to some values. However, more samples also imply an increase in memory and computational time for each sensor node. At the beginning, when the $N$ is increased from 1 to 5 , there is a significant reduction in the estimation error. This is because a small number of samples cannot correctly approximate the probability distribution of the node's location. For all three MCL algorithms, the estimation error reaches the steady when $N$ is greater than 30 .

Fig. 6 shows the estimation error by increasing the maximum velocity of a node or seed. The velocity of a node or seed is uniformly distributed between 0 and $v_{\max }$ and a fixed paused time of 5 time units is considered. Although the location information may become obsolete when nodes and seeds are moving, the sensor node has a higher chance to obtain new information from the seeds than it has encountered.

Results from Figs. 1-6 consider an idealized radio model. We also use the technique in [4] to model the irregular radio pattern. In this model, all nodes within half of the maximum transmit radio range of anchors are guaranteed to hear from the anchor, whereas nodes between the maximum radio range and half of that range may or may not hear from the anchor depending on the radio pattern in that direction. The degree of 


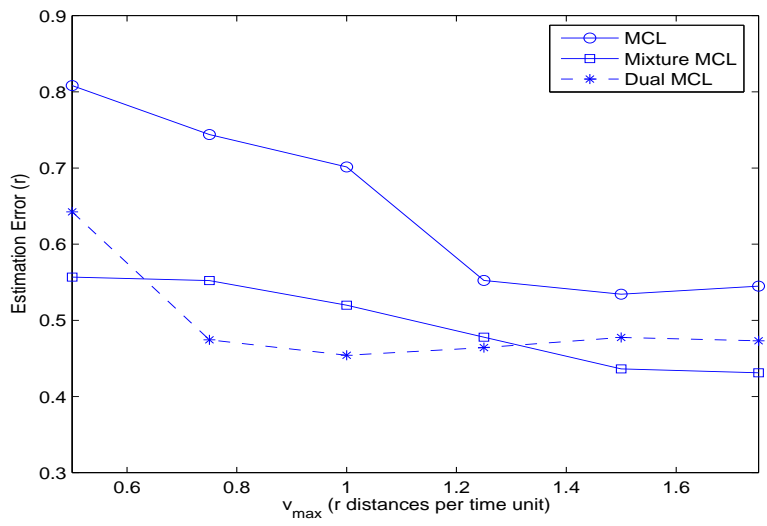

Fig. 6. Estimation error versus maximum velocity with $n_{d}=10, s_{d}=2$, $N=35, K=5$ and $\phi=0.2$.

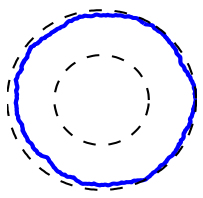

$\mathrm{DOI}=0.01$

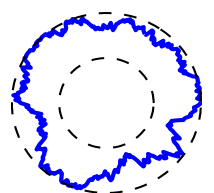

$\mathrm{DOI}=0.05$

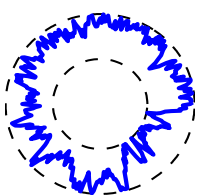

$\mathrm{DOI}=0.10$
Fig. 7. Irregular radio patterns for different values of DOI.

irregularity (DOI) parameter is defined as the maximum radio range variation per unit degree change in direction. Examples of different DOI values of this irregular radio pattern model are shown in Fig. 7. Fig. 8 shows the estimation error versus the DOI. Both dual and mixture MCL algorithms are not significantly affected by changes in the DOI. However, more time is required to obtain the samples for these algorithms.

Although the dual MCL algorithm outperforms the original and mixture MCL algorithms in terms of a lower estimation error, it is important to note that the dual sampling methodology requires more time to obtain samples from the sensors (i.e., from (2)-(4)). This time varies according to the different values of parameters considered (e.g., $v_{\max }$, DOI). Among the three MCL algorithms that we study in this paper, the mixture MCL algorithm appears to be a good trade off between computational time and location accuracy.

\section{CONCLUSIONS}

In this paper, we proposed and analyzed the dual and mixture MCL algorithms for localization in mobile WSN. Simulation experiments are conducted to evaluate the performance by varying several parameters including the node and seed densities, number of samples, velocity of nodes, and DOI values. Results show both dual and mixture MCL algorithms are able to reduce the estimation error under a wide range of conditions when compared with the original MCL algorithm. Due to an increase in time required to obtain samples in the dual MCL sampling methodology, the mixture MCL algorithm is shown to be a good trade off in terms of computational time and location accuracy.

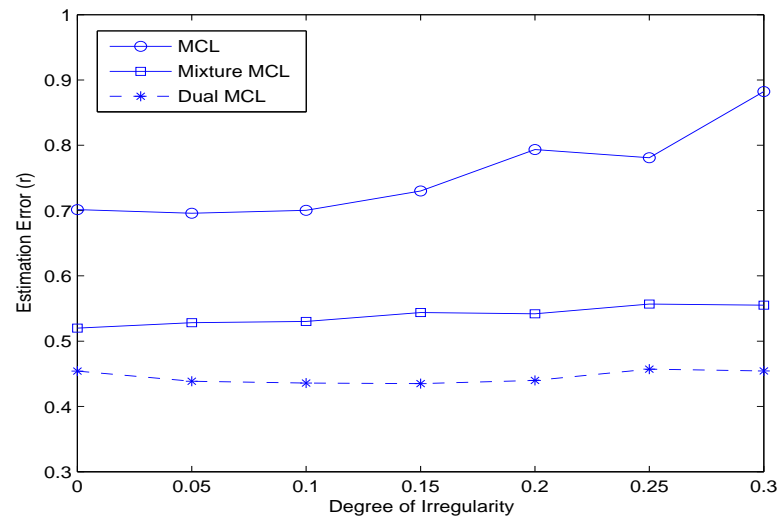

Fig. 8. Estimation error versus degree of irregularity with $n_{d}=10, s_{d}=2$, $v_{\max }=r, N=35, K=5$ and $\phi=0.2$.

\section{ACKNOWLEDGMENT}

This work is supported by the Natural Sciences and Engineering Research Council (NSERC) of Canada and the Programa de Mejoramiento del Profesorado (PROMEP) from Mexico.

\section{REFERENCES}

[1] D. Niculescu, "Positioning in ad hoc sensor networks," IEEE Network Magazine, vol. 18, pp. 24-29, July 2004.

[2] Y. Shang, W. Ruml, Y. Zhang, and M. Fromherz, "Localization from mere connectivity," in Proc. of ACM MobiHoc, Annapolis, MD, June 2003.

[3] L. Doherty, K. Pister, and L. Ghaoui, "Convex position estimation in wireless sensor networks," in Proc. of IEEE Infocom, Anchorage, AK, April 2001.

[4] T. He, C. Huang, B. Lum, J. Stankovic, and T. Adelzaher, "Range-free localization schemes for large scale sensor networks," in Proc. of ACM MobiCom, San Diego, CA, Sept. 2003.

[5] V. Vivekanandan and V. Wong, "Concentric anchor-beacons (CAB) localization for wireless sensor networks," in Proc. of IEEE ICC, Istanbul, Turkey, June 2006.

[6] Y. Shang, W. Ruml, and Y. Zhang, "Improved MDS-based localization," in Proc. IEEE Infocom, Hong Kong, China, March 2004.

[7] D. Niculescu and B. Nath, "Ad-hoc positioning system," in Proc. of IEEE Globecom, San Antonio, TX, Nov. 2001.

[8] K. Chintalapudi, A. Dhariwal, R. Govindan, and G. Sukhatme, "Ad-hoc localization using ranging and sectoring," in Proc. of IEEE Infocom, Hong Kong, China, March 2004.

[9] M. Sichitiu and V. Ramadurai, "Localization of wireless sensor networks with a mobile beacon," in Proc. of IEEE MASS, Fort Lauderdale, FL, October 2004.

[10] K. F. Ssu, C. H. Ou, and H. Jiau, "Localization with mobile anchor points in wireless sensor networks," IEEE Trans. on Vehicular Technology, vol. 54, pp. 1186-1197, May 2005.

[11] P. Pathirana, N. Bulusu, A. Savkin, and S. Jha, "Node localization using mobile robots in delay-tolerant sensor networks," IEEE Trans. on Mobile Computing, vol. 4, pp. 285-296, July/Aug 2005.

[12] L. Hu and D. Evans, "Localization for mobile sensor networks," in Proc of ACM MobiCom'04, Sept. 2004.

[13] N. Bulusu, J. Heidemann, and D. Estrin, "GPS-less low-cost outdoor localization for very small devices," IEEE Personal Communications, vol. 7, no. 5, pp. 28-34, October 2000.

[14] R. Nagpal, H. Shrobe, and J. Bachrach, "Organizing a global coordinate system from local information on an ad hoc sensor network," in Proc. of IPSN'03, April 2003.

[15] S. Thrun, D. Fox, W. Bugard, and F. Dellaert, "Robust Monte Carlo localization for mobile robots," Artificial Intelligence, vol. 128, pp. 99 $141,2001$.

[16] http://www.cs.virginia.edu/mcl. 